\title{
Optical radiomic signatures derived from OCT images to improve identification of melanoma
}

\author{
Turani, Zahra ; Fatemizadeh, Emad ; Blumetti, Tatiana ; Daveluy, Steven ; Moraes, Ana Flavia ; Chen, \\ Wei ; Mehregan, Darius ; Andersen, Peter E.; Nasiriavanaki, Mohammadreza
}

\section{Published in:}

Proceedings of SPIE

Link to article, DOI:

$10.1117 / 12.2526624$

Publication date:

2019

Document Version

Publisher's PDF, also known as Version of record

Link back to DTU Orbit

Citation (APA):

Turani, Z., Fatemizadeh, E., Blumetti, T., Daveluy, S., Moraes, A. F., Chen, W., Mehregan, D., Andersen, P. E., \& Nasiriavanaki, M. (2019). Optical radiomic signatures derived from OCT images to improve identification of melanoma. In Proceedings of SPIE: Optical Coherence Imaging Techniques and Imaging in Scattering Media III [110780O] Proceedings of SPIE - The International Society for Optical Engineering https://doi.org/10.1117/12.2526624

\section{General rights}

Copyright and moral rights for the publications made accessible in the public portal are retained by the authors and/or other copyright owners and it is a condition of accessing publications that users recognise and abide by the legal requirements associated with these rights.

- Users may download and print one copy of any publication from the public portal for the purpose of private study or research.

- You may not further distribute the material or use it for any profit-making activity or commercial gain

- You may freely distribute the URL identifying the publication in the public portal 


\section{Optical radiomic signatures derived from OCT images to improve identification of melanoma}

\section{Zahra Turani, Emad Fatemizadeh, Tatiana Blumetti, Steven Daveluy, Ana Flavia Moraes, et al.}

Zahra Turani, Emad Fatemizadeh, Tatiana Blumetti, Steven Daveluy, Ana Flavia Moraes, Wei Chen, Darius Mehregan, Peter E. Andersen, Mohammadreza Nasiriavanaki, "Optical radiomic signatures derived from OCT images to improve identification of melanoma," Proc. SPIE 11078, Optical Coherence Imaging Techniques and Imaging in Scattering Media III, 1107800 (19 July 2019); doi: 10.1117/12.2526624 


\title{
Optical Radiomic Signatures Derived from OCT Images to Improve Identification of Melanoma
}

\author{
Zahra Turani $^{1,2}$, Emad Fatemizadeh ${ }^{1}$, Tatiana Blumetti ${ }^{3}$, Steven Daveluy ${ }^{4}$, Ana Flavia Moraes ${ }^{3}$, Wei \\ Chen $^{5}$, Darius Mehregan ${ }^{3}$, Peter E. Andersen ${ }^{6(*)}$, and Mohammadreza Nasiriavanaki ${ }^{2,4,5(* *)}$ \\ ${ }^{1}$ Department of Electrical Engineering, Sharif University of Technology, Tehran, Iran \\ ${ }^{2}$ Department of Biomedical Engineering, Wayne State University, Detroit, MI \\ ${ }^{3}$ Cutaneous Oncology Department, AC Camargo Cancer Center, São Paulo, Brazil \\ ${ }^{4}$ Department of Dermatology, School of Medicine Wayne State University \\ ${ }^{5}$ Department of Oncology, Karmanos Cancer Institute, Detroit, MI \\ ${ }^{6}$ Department of Health Technology, Technical University of Denmark, Denmark \\ ${ }^{(*)}$ Corresponding author: Peter E. Andersen (peta@dtu.dk) \\ ${ }^{(* *)}$ Corresponding author: Mohammadreza Nasiriavanaki (mrn.avanaki@wayne.edu)
}

\begin{abstract}
Malignant melanoma is by far the most dangerous type of skin cancer. Currently, the gold standard to diagnose melanoma in the clinic is excisional biopsy and histopathologic analysis. Approximately 15-30 benign lesions are biopsied to diagnose each melanoma. Additionally, biopsies are invasive and result in pain, anxiety, scarring and disfigurement of patients, and they can be a financial burden to the health care system. Among several imaging techniques developed to enhance melanoma diagnosis, optical coherence tomography (OCT) with its high-resolution and intermediate penetration depth can potentially provide required diagnostic information, noninvasively. We propose an image analysis algorithm, 'optical properties extraction (OPE)' that drastically improves the specificity and sensitivity of OCT by identifying unique optical radiomic signatures pertinent to melanoma detection. We evaluate the performance of the algorithm using several tissue-mimicking phantoms. We then test the OPE algorithm with sixty-nine human subjects and demonstrate that melanoma can be differentiated from benign nevi with $97 \%$ sensitivity, and $98 \%$ specificity.
\end{abstract}

Introduction. Malignant melanoma is by far the most dangerous type of skin cancer [1, 2]. The initial step in a physician's decision to biopsy a suspicious lesion is dermoscopic inspection using the ABCDE criteria [3]. A lesion that apparently fulfills the ABCDE criteria for melanoma is biopsied for definitive histopathologic diagnosis [3]. Several non-invasive imaging approaches have been developed for the diagnosis of melanoma and differentiation from benign nevi. Their clinical utility, however, is limited because they do not provide sufficient specificity and sensitivity.

Methodology. Tissues have intrinsic scattering characteristics based on the density, size and shape of tissue microstructures; absorption characteristics derived from chromophore concentration; and, anisotropy factor which correlates to cell size and disorder. These characteristics are modified during tumor development. Methods that can uniquely identify these characteristics hold promise for providing diagnostic value [4]. OCT is inherently sensitive to the changes of these characteristics [5]. However, its sensitivity and specificity in differentiating morphologically similar structures is low, due to the interrelationships of these optical characteristics. Previously, we explored texture analysis of OCT images, but the improvement was limited [6]. Here we propose an image analysis algorithm based on the EHF principle, which we call OPE, to disaggregate the OCT image into its individual optical attributes. These optical attributes, when extracted from the OCT image form a set of tissue specific optical radiomic features. The method presented here demonstrates significant improvements in melanoma detection over current clinical methods.

The method of the optical radiomic melanoma detection (ORMD) protocol can be outlined in the following steps: 1) acquisition of OCT images from suspected and nearby healthy skin; 2) the OCT B-scans 
are pre-processed (e.g., averaged) and a region of interest (ROI) selected; 3) optical properties are extracted by fitting the EHF model to the intensity profile of the averaged A-line obtained from the ROI yielding the scattering coefficient, absorption coefficient and anisotropy factor from the suspected lesion (we call it signal) and nearby healthy (we call it control) skin to create a set of normalized optical radiomic features; and 4) the selected optical radiomic features, i.e., optical radiomic signatures, with the optimum classifier render the status of the tissue: "Tissue sample is consistent with healthy tissue," or "Tissue sample exhibits characteristics consistent with melanoma.”

Results. Our initial validation was conducted on milk and milk-ink phantoms, to determine if the OPE algorithm correctly correlated to changes in optical properties of the phantoms, specifically, scattering and absorption coefficients. We observed the following in the OPE-extracted optical properties: the scattering coefficient $\left(\mu_{s}\right)$ progressed almost linearly with increasing milk concentration $(p<0.001)$; the absorption coefficient $\left(\mu_{\mathrm{a}}\right)$ in milk phantoms progressed almost linearly with increasing the milk concentration $(p$ $<0.001$ ); the absorption coefficient in milk-ink phantoms progressed almost linearly with increasing the ink concentration $(p<0.01)$. Also we observed that the OPE-extracted $\mu_{\mathrm{s}}$ in milk-ink phantoms shows slight variation ( $p<0.001$ with $\Delta=1\left[\mathrm{~mm}^{-1}\right]$ ). The values of anisotropy factor $(g)$ show slight variation in both milk and milk-ink phantoms ( $p<0.05$ with $\Delta=0.03$ ), which is consistent with the phantoms being homogenous solutions consisting of scatterers of near identical size. The $\Delta$ values were chosen empirically. The average fitting error in both datasets was about $4 \%$. Precision of the obtained values can be improved by using a higher resolution OCT.

The next tests were conducted on human subjects. Our method is non-invasive, the OCT system used is FDA approved, and we acquired IRB approval for testing on human subjects. Sixty-nine melanoma, benign nevi and healthy subjects were recruited. The results obtained from the clinically identified melanoma to non-melanoma area showed a meaningful difference. Differences due to factors such as skin type, ethnicity, sun exposure, etc., were negated when normalized to nearby healthy skin. The large standard deviation of the optical radiomic features for melanoma images correlates to irregularity in tissue structure; signifying disease. Our results were consistent with the finding that the scattering and absorption coefficients increase with the concentration of melanocytes (melanocyte frequency - melanoma: $71 \pm 11 \%$; benign nevi: $18 \pm 3 \%$; healthy: $14 \pm 3 \%$ ); anisotropy factor increased with cell size (average mean diameter of 200 consecutive melanocytes - melanoma: $16 \pm 3 \mu \mathrm{m}$; benign nevi: $7 \pm 0.4 \mu \mathrm{m}$; healthy: $6 \pm 0.4 \mu \mathrm{m}$ ) and tissue disorder, due to cellular displacement. This was also observed in simulations using the OMLC online Mie calculator [7].

We propose that increase in scattering and absorption coefficients, respectively, may be due to increased concentration of melanocytes, and the increase in anisotropy factor may be due to increased cell size. The combination of increased numbers of melanocytes that are larger with pleomorphic nuclei is the hallmark of melanoma on pathological assessment [8].

Six optical radiomic features are generated by the OPE algorithm from the OCT images. These are the mean and standard deviation of scattering coefficient, absorption coefficient and anisotropy factor. As there are only six features, we were able to examine each possible combination of features to identify the optimal feature set [9]. This exhaustive search reaches the optimal feature sets, because it systematically enumerates all possible candidates, it finds the optimal feature set more efficiently, compared to other similar methods designed for feature selection, such as sequential floating forward search (SFFS) and sequential floating backward search (SFBS) [9].

As for the criteria to choose the most appropriate classifier, we need to estimate the true class probability density function (pdf) [9]. With small to medium size datasets, such a function cannot accurately be estimated, and the performance of the classifiers is difficult to calculate. As a rule of thumb, low variance classifiers (i.e., Naïve Bayes, SVM) are preferred for such datasets. The recommended method in this case 
is to find best classifier with the aid of validation/training and a repeated random sampling strategy [10]. We selected six established classifiers, each was trained and tested on the data using a 20-fold cross validation algorithm; this evaluates the classifier generalization. Values for sensitivity, specificity, Jaccard index and accuracy, were determined by testing all permutations of the six features, in combination with each classifier. Based on clinical requirements of high specificity and sensitivity, a specific classifier and set of features were selected. Some combinations generated high sensitivity with low specificity, or vice versa. For example, features $\mathrm{F}_{2}, \mathrm{~F}_{3}$, with the GSVM $(\mathrm{C}=1)$ classifier resulted in the best sensitivity (99\%) with a specificity of $50 \%$. The best overall was a combination of features $\mathrm{F}_{1}$ through $\mathrm{F}_{5}$ with the GSVM classifier; results were sensitivity $(97 \% \pm 3 \%)$, specificity $(98 \% \pm 2 \%)$, Jaccard index (93\% $\pm 5 \%)$, and accuracy $(98 \% \pm 2 \%)$. For the preferred classifier, GSVM, we calculated the area under the curve (AUC) with different $\mathrm{C}$-values, and $\mathrm{C}=2.1$ gave us the best results.

Using a computer with a Core i7 CPU and 8 GB memory, with MATLAB2015a, it took 1.2 seconds to assess a suspected lesion with input of OCT images from healthy and suspect tissue. Using lower level programming languages e.g. $\mathrm{C}$ and parallel processing, the computation time will greatly be improved.

Comparing the optical radiomic melanoma detection (ORMD) methodology to an established noninvasive clinical examination procedure, a dermoscopic analysis was made using the Two-Step algorithm followed by Pattern Analysis [11, 12]. Two experienced dermatologists, Dr. Tatiana Blumetti, M.D., and Dr. Ana Moraes, M.D., from dermatology clinic at the Skin Cancer Department in AC Camargo Cancer Center in Brazil performed the dermoscopic analysis. The suspicious lesions were selected based on changes on dermoscopic follow-up. Assessment of dermoscopy images compared to results of the ORMD methodology, showed a significant diagnostic improvement. Using ORMD only one unnecessary biopsy for melanoma was performed, while dermoscopy identified 10 benign nevi as possible melanoma, necessitating 10 biopsies. In melanoma, OPE missed one case, where dermoscopy misdiagnosed four cases as benign nevi, resulting in delayed treatment. In all cases, the tissue statuses were confirmed by histological analysis.

Conclusion. The statistics indicate that ORMD-based diagnosis is reliable and can effectively differentiate between melanoma and non-melanoma cases. Still, a larger number of subjects is required to make a more rigorous conclusion. Overall, the rate of unnecessary biopsies is significantly decreased with the use of the ORMD methodology. A larger number of subjects may necessitate the use of a more sophisticated classification algorithm which may further increase the accuracy of the ORMD methodology and minimize the number of misdiagnoses. The next practical challenge for the proposed method is the development of a real-time, 3-D melanoma margin detection algorithm for use during biopsy procedures for guidance.

\section{References.}

1. Linos, E., et al., Increasing burden of melanoma in the United States. Journal of Investigative Dermatology, 2009. 129(7): p. 1666-1674.

2. Bharath, A. and R. Turner, Impact of climate change on skin cancer. Journal of the Royal Society of Medicine, 2009. 102(6): p. 215-218.

3. Friedman, R.J., D.S. Rigel, and A.W. Kopf, Early detection of malignant melanoma: The role of physician examination and self-examination of the skin. CA: a cancer journal for clinicians, 1985. 35(3): p. 130-151.

4. D. Levitz, et al., Determination of optical scattering properties of highly-scattering media in optical coherence tomography images, Optics Express 2004. 12:pp. 249-259.

5. $\quad$ Turchin, I.V., et al., Novel algorithm of processing optical coherence tomography images for differentiation of biological tissue pathologies. Journal of biomedical optics, 2005. 10(6): p. 064024.

6. $\quad$ Adabi, S., et al., Universal in vivo Textural Model for Human Skin based on Optical Coherence Tomograms. Scientific reports, 2017. 7(1): p. 17912.

7. Available from: http://omlc.org/calc/mie_calc.html.

8. $\quad$ Rhodes, A.R., et al., Increased intraepidermal melanocyte frequency and size in dysplastic melanocytic nevi and cutaneous melanoma. A comparative quantitative study of dysplastic melanocytic nevi, superficial spreading melanoma, nevocellular nevi, and solar lentigines. Journal of Investigative Dermatology, 1983. 80(5): p. 452-459.

9. Theodoridis, S. and K. Koutroumbas, Pattern recognition. 2009. Elsevier Inc. 
10. Basavanhally, A., S. Viswanath, and A. Madabhushi, Predicting classifier performance with limited training data: applications to computer-aided diagnosis in breast and prostate cancer. PloS one, 2015. 10(5): p. e0117900.

11. Braun, R.P., et al., Pattern analysis: a two-step procedure for the dermoscopic diagnosis of melanoma. Clinics in dermatology, 2002. 20(3): p. 236-239.

12. Marghoob, A.A., et al., The beauty and the beast sign in dermoscopy. Dermatologic Surgery, 2007. 33(11): p. 13881391. 